\title{
POST-AGB CANDIDATES
}

\author{
L.B.F.M. WATERS \\ Astronomical Institute Anton Pannekoek, University of Amsterdam, Kruislaan 403, 1098 \\ SJ Amsterdam \\ SRON Laboratory for Space Research Groningen, P.O. Box 800, 9700 AV Groningen \\ and \\ K.C. SAHU \\ Kapteyn Astronomical Institute, P.O. Box 800, 9700 AV Groningen
}

\begin{abstract}
A review is given of the properties of post-AGB stars. Methods to find candidate post-AGB stars are briefly discussed, along with some results and selection effects involved. Their photospheric parameters $\left(\mathrm{T}_{\text {ef } f}, \log \mathrm{g}\right.$, abundances), energy distributions and binarity are also discussed. Evidence for mass loss, both past and present, is reviewed.
\end{abstract}

Key words: post-AGB stars - mass loss - evolution - binarity

\section{Introduction}

The transition of low and intermediate mass stars from the Asymptotic Giant Branch (AGB) into a Planetary Nebula (PN) is one of the shortest phases in the evolution of such stars, but nevertheless is of importance for a better understanding of the formation mechanism of PNe and of the way the star leaves the AGB. Observations play a dominant role in attempts to improve our knowledge, because the theory of stellar evolution cannot predict the course of events from first principles. This review describes our current understanding of the physics of transition objects: several methods to find post-AGB stars, their photospheric properties and the circumstellar environment. The binary nature of some well-known post-AGB stars is discussed and the effect of binarity on the geometry of the circumstellar material, and on the photospheric abundance patterns. For other recent reviews on this subject we refer to Waters et al. (1992) and Waelkens \& Waters (1992).

\section{Selection of post-AGB candidates}

Three methods are used in the literature to find post-AGB stars: searches of the IRAS colour-colour diagram, correlations of stellar catalogues with the IRAS database, and the study of luminous stars far from the galactic plane. All three methods have severe selection effects and will result in finding a limited subset of post-AGB stars.

The most widely used method is based on the change of the far-IR colours of AGB stars as they move off the AGB towards higher effective temperature. The remnant of the AGB wind will cool and become transparent at near-IR and optical wavelengths, while the IRAS colours of the star become redder. It was noticed by Pottasch et al. (1988), and also by van der Veen et al. (1989), that such a relation does indeed exist in the IRAS color-color diagram. In this diagram, the red stars are found mostly around the black body curve of $500 \mathrm{~K}$ while the PN are found to have an effective temperature of 100 to $200 \mathrm{~K}$. The $\mathrm{OH} / \mathrm{IR}$ stars, thought to be

R. Weinberger and A. Acker (eds.), Planetary Nebulae, 271-278.

(C) 1993 IAU. Printed in the Netherlands. 


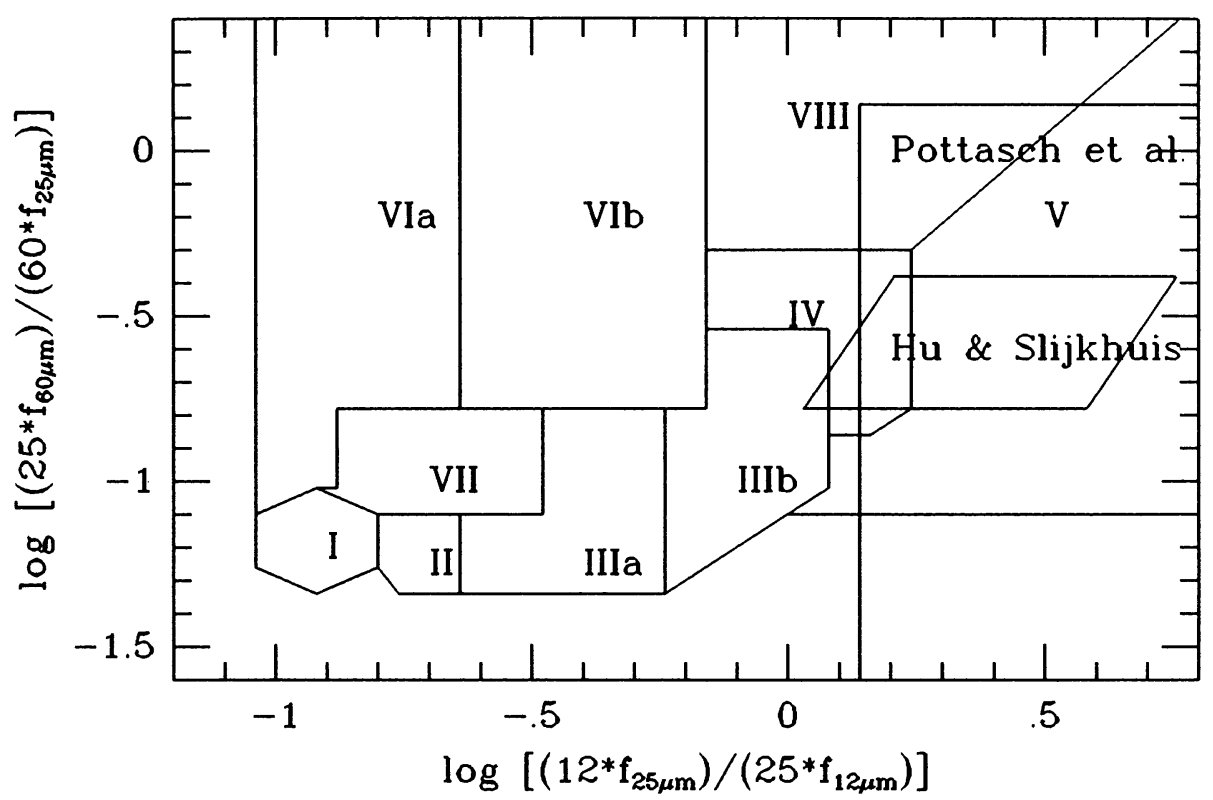

Fig. 1. The IRAS colour-colour diagram with the different regions corresponding to various classes of objects (van der Veen and Habing, 1989). The colour criteria used by $\mathrm{Hu}$ and Slijkhuis is shown by a trapezium and the rather relaxed criteria used by Pottasch et al. is shown by a bigger rectangle.

in the transition phase between the two stages, fall between the two temperature ranges as can be seen from Fig. 1. This roughly corresponds to the region $\mathrm{V}$ of van der Veen et al. (1989). So this method of looking at a fixed region of the colorcolor diagram has been employed by various groups, the details of which are briefly described below.

Hu and Slijkhuis $(1991,1992)$ have aimed at the follow-up multi-frequency study of a complete sample of post-AGB candidates from the IRAS catalog, in order to determine the nature and properties of these sources. To keep the size of the sample reasonable, they have however used rather stringent criteria, which is represented by the rectangular box shown in Fig. 1. A further criterion used in their sample is that the 12 micron flux is larger than $2 \mathrm{Jy}$, which makes the sample volume limited. About $30 \%$ of the known PNe lie in their colour-colour box. Out of the 98 sources that satisfy these criteria, 42 are southern sources. 23 out of these have no optical counterparts in the ESO survey films and the rest have been taken up for further study in the optical region by obtaining spectra and images. 17 are identified as bright stars which have spectral types F, G and M.

The approach of Pottasch and co-workers (Pottasch et al. 1988; Manchado et al. 1989 a,b; Sahu et al. 1992; Garcia-Lario, 1991) has been to use rather relaxed 
colour criteria to select post-AGB and PN candidates from the IRAS catalog. The criteria used by them, the details of which are given by Pottasch et al. (1988), is given by: $f_{12 \mu m} / f_{25 \mu m}<0.35$ and $f_{25 \mu m} / f_{60 \mu m}>0.3$ (shown by the bigger box in Fig. 1), and good IRAS flux quality at 12 and 25 microns. (The flux quality at 60 micron need not be high since lower quality flux in IRAS catalog only means an upper limit, and hence the criteria still hold). The aim of this has been not only to pick up post-AGB stars but also new PN's, although the method more efficiently picks up the dusty and hence younger PN. This criterion gives a sample of $\sim 1000$ sources, 500 of which are known PNe. The rest have been taken up for further optical, near-IR and radio continuum study.

Out of the $\sim 200$ sources in the sample of Pottasch that are observed in the optical and near-IR region so far, about 70 are $\mathrm{OH} / \mathrm{IR}$ stars 35 of which are nonvariable. About 35 are new or possible $\mathrm{PN}$, and about a similar number are probable post-AGB stars many of which show $\mathrm{H} \alpha$ line emission. The rest are still unknown, the nature of which are currently under investigation.

The objects observed so far have some selection effects. The ones that can be more easily seen on the Palomar plates seem to have been preferentially taken up for optical study. For a small fraction of them, particularly in a crowded field close to the galactic plane, the object observed may not be the correct IRAS counterpart although the objects which show post-AGB nature are almost certainly the correct counterparts. In this project, the objects that do not show any post-AGB nature are being re-observed by taking images in $\mathrm{H} \alpha$ and other bands. These images will be analysed to check whether there is any object in the IRAS uncertainty ellipse that shows post-AGB characteristics. For example, if there is some object in the IRAS box which shows enhanced $\mathrm{H} \alpha$ emission, that may be the correct counterpart, the spectrum will then be taken to confirm the nature.

A specially interesting example of this sample is IRAS 06562-0337 in terms of its fast evolution (Garcia-Lario et al. 1992). A spectrum of this object taken in 1987 shows mainly the hydrogen lines in emission (Fig. 2). A spectrum obtained about 2 years later shows many more emission lines including the forbidden lines [OIII] $\lambda \lambda 4959,5007 \AA$, characteristic of a high density (as derived from the line ratios), young planetary nebula. The increase in intensity of the He I $\lambda 5876 \AA$ line during this period, and the change in the $\mathrm{H} \beta$ to continuum ratio both indicate an increase of stellar temperature. The object also shows strong CO emission in millimetric wavelengths. A spectrum obtained in 1992 however shows that the forbidden lines have all disappeared. Since the spectrum obtained in 1988 shows evidence of mass loss in the form of a P-Cygni profile, the authors have interpreted this as the final mass loss episode which precedes the formation of a planetary nebula. If this interpretation is correct, the object should soon show the forbidden lines in emission again, and monitoring this object may provide an opportunity to follow the evolution to a PN.

Similar work by others (e.g. Kwok et al. 1987; Parthasarathy \& Pottasch 1986; Hrivnak et al. 1988, 1989; van der Veen et al. 1989; Kwok 1992) also shows that many post-AGB stars can be found in the IRAS colour-colour diagram in the region between those of the reddest AGB stars and the locus of the PNe. These searches yield objects that are bright in the IR, i.e. have large amounts of circumstellar dust: 
IRAS $06562-0337$

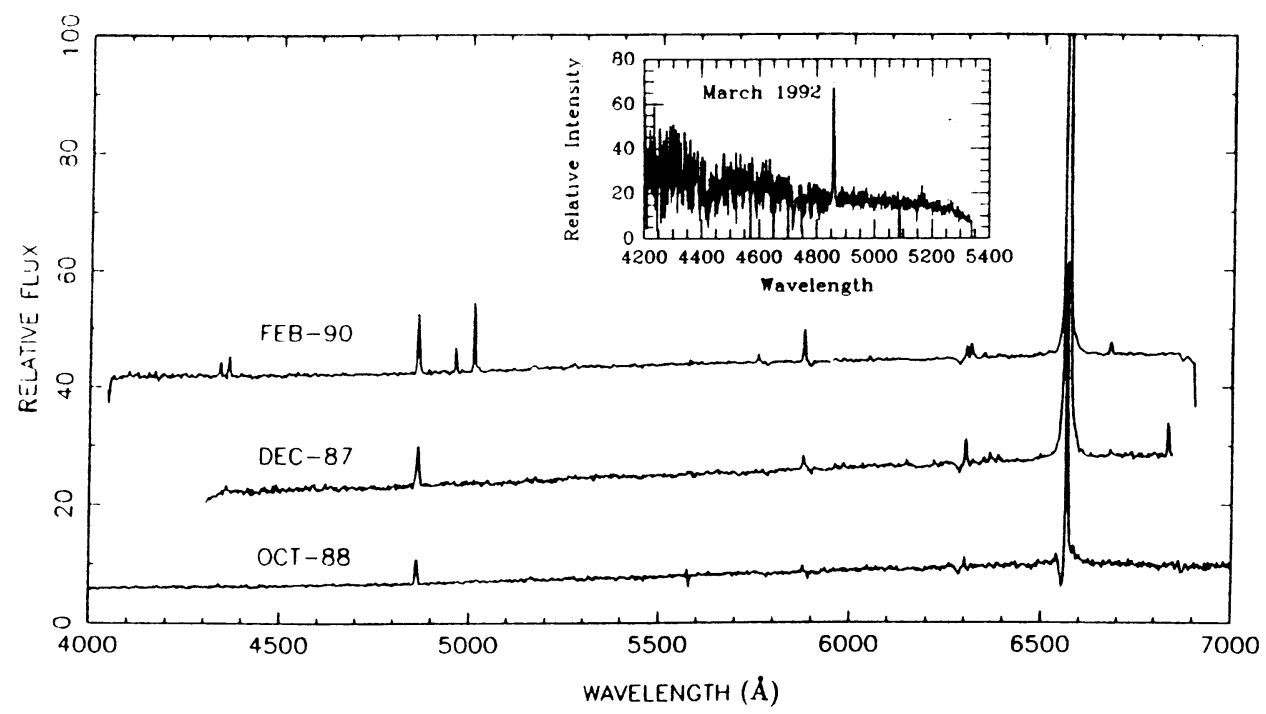

Fig. 2. The optical spectra of IRAS 06562-0337 obtained at different times (Garcia-Lario et al. 1992). Notice the onset of forbidden line emissions in 1990, and the significant increase of the He I $\lambda 5976 \AA$ line, indicating the increase in the intensity of stellar temperature.

$\mathrm{L}_{I R}>>\mathrm{L}_{\text {opt }}$. Therefore preferentially the more massive objects will be found.

The cross-correlation of optical catalogues with the IRAS database also has been successful (see e.g. Lamers et al. 1986; Waelkens et al. 1987; Parthasaraty \& Pottasch 1988; Trams et al. 1990; Waelkens et al. 1990; Stencel \& Backman 1991; Oudmaijer et al. 1993). No restriction on the IRAS colours is used, and so confusion with other kinds of stars with circumstellar dust is a problem. The study of Oudmaijer et al. (1993) of the correlation between the SAO catalogue and the IRAS PSC not only resulted in the finding of new post-AGB stars, but also yielded some pre-main-sequence objects as well as Vega-type stars. Detailed study of individual objects is necessary to establish their nature. The use of the SAO catalogue introduces a bias towards objects that have small amounts of dust or that have non-spherically symmetric dust envelopes. In most cases $\mathrm{L}_{I R} \approx \mathrm{L}_{\text {opt }}$.

The third approach is used by e.g. de Boer and co-workers and Dufton and collaborators. They study the properties of faint blue stars in the halo of the Galaxy by means of abundance analyses, and find objects with Fe deficiencies and CNO enhancements (Conlon et al. 1991; de Boer et al. 1992). In one case a low-excitation PN was found around one of the halo B stars (Conlon et al. 1992), which is also an IRAS source. No other star was found in the IRAS PSC (Conlon, private communication), so $\mathrm{L}_{I R}<<\mathrm{L}_{\text {opt }}$.

It is interesting to note that most searches for post-AGB stars have yielded stars with spectral type $F$ or $G$, irrespective of the method used (apart from the halo $\mathrm{B}$ stars). Two different effects may play a role in preferentially finding $\mathrm{F}$ and $\mathrm{G}$ 
type objects: (1) for low mass stars, the evolution to higher $\mathrm{T}_{\text {eff }}$ is so slow that by the time they reach spectral type A or B, the dust shell has already cooled to flux levels below the IRAS detection limit. (2) for more massive objects, the evolution from $\mathrm{G}$ to $\mathrm{F}$ roughly takes 1000 years (e.g. Schönberner 1983), but after that $\mathrm{T}_{\text {eff }}$ increases much more rapidly (Oudmaijer et al. 1992).

Since detailed optical studies of faint post-AGB stars have not (yet) been carried out, most of our knowledge of their properties stems from studies of objects that are optically bright. As mentioned above, the bright post-AGB stars may not be a representative sample because of the bias towards low amounts of circumstellar dust.

\section{Radial velocity variations: pulsation and binarity}

For bright post-AGB stars accurate radial velocities (RV) can be measured and thus also RV variations. Such studies have revealed that the UU Her stars show RV variations that can be explained in terms of pulsations (see e.g. Sasselov, these proceedings). Typical amplitudes are a few $\mathrm{km} / \mathrm{s}$ and pulsation periods are of the order of 60 to 80 days. A systematic study of RV variations for a large sample of post-AGB candidate stars found in the IRAS PSC is being carried out by Waelkens and Mayor (1992) using CORAVEL. Preliminary results of this study indicate that most stars show evidence for RV variations. A significant fraction however shows RV variations with an amplitude and period that cannot be explained by pulsation, but must be due to orbital motions. Most orbital periods found are of the order of 100 to several hundred days, but longer orbital periods cannot be excluded at present due to the limited time coverage. Examples of binary post-AGB stars are HR 4049 (Waelkens et al. 1991), 89 Her (Arrelano-Ferro 1984; Waters et al. 1993) and HD 46703 (Waelkens \& Mayor 1992).

The binary nature of post-AGB stars has interesting implications for their evolution: the orbital parameters found in e.g. $89 \mathrm{Her}$ and HR 4049 suggest that these stars filled their Roche lobe on the AGB, and would have experienced a common envelope phase. It is not clear how these systems could have survived such a phase, especially in view of the (significant) non-zero eccentricities that are found: a common envelope phase tends to rapidly circularize the orbit.

\section{Binarity and hot dust}

The IR energy distribution of post-AGB stars not only shows evidence for cool dust $(\mathrm{T} \approx 100-200 \mathrm{~K})$ but in some stars hot dust $(\mathrm{T} \approx 500-1300 \mathrm{~K})$ also is found (Trams et al. 1989). The cool dust usually is explained in terms of a detached dust shell expelled while the star was on the AGB, but the hot dust must be very close to the star and have been ejected very recently. Trams et al. (1989) interpreted this as strong evidence for significant post-AGB mass loss. More detailed study of the stars with hot dust shells shows that they are binaries (Waters et al. 1991), which suggests that the presence of a companion triggers the presence of hot dust. In HR 4049 there is evidence that the hot dust is in a disc around the system (Waelkens et al. 1991); this model is also applicable to 89 Her (Waters et al. 1993). 
TABLE I

Basic properties of some stars with depleted atmospheres

\begin{tabular}{ccccccccc}
\hline HD & name & Sp. t. & $\mathrm{T}_{\text {eff }}$ & $\log \mathrm{g}$ & dust & {$[\mathrm{Fe} / \mathrm{H}]$} & {$[\mathrm{S} / \mathrm{Fe}]$} & $\log (\mathrm{C} / \mathrm{O})$ \\
\hline 89353 & HR4049 & F I & 7500 & 1.0 & yes & -4.8 & +4.4 & -0.23 \\
52961 & & G : & 6500 & 1.0 & yes & -4.8 & +3.7 & -0.25 \\
44179 & Red Rectangle & F I & 7500 & 1.0 & yes & -3.5 & +3.3 & -0.08 \\
& BD+39 4926 & F I & 7500 & 1.0 & no & -2.9 & +3.0 & -0.60 \\
46703 & & F I & 6000 & 0.4 & yes & -1.6 & +1.2 & -0.24 \\
\hline
\end{tabular}

Note to Table 1: data taken from Waelkens et al. 1992 and references therein

If the hot dust is in orbit around the system rather than in an outflow, it may be the remnant of a disc formed while the star was still on the AGB. In this case the temperature of the hot dust is not a measure of the time that has elapsed since it was ejected, but merely reflects the distance between the star and the inner radius of the circum-system disc.

The presence of hot dust which is 'trapped' in a binary system is not restricted to post-AGB stars. The C-rich AGB stars with O-rich dust shells may also be binaries in which the O-rich dust was ejected by the AGB star when it was still an $M$ star. The dust either is in a circum-system disc or in a disc around the (unseen) companion (Morris 1989). Other examples are the massive binary 3 Puppis, and the interacting binary $v \mathrm{Sgr}$, showing that the mechanism that creates such discs also operates in massive Pop. I stars.

\section{Chemical abundances}

Several post-AGB stars have been the subject of detailed abundance determinations (for a recent review see Bond 1991). These studies have shown that there is a wide range in metallicities, from $[\mathrm{Fe} / \mathrm{H}] \approx-5.0$ to 0.0 ! The stars with extremely low metallicities (e.g. HR 4049, HD 52961) show remarkable abundance patterns that cannot be explained by nucleosynthesis: their $\mathrm{C}, \mathrm{N}, \mathrm{O}$, and $\mathrm{S}$ abundance is solar within an order of magnitude, but many metals ( $\mathrm{Fe}, \mathrm{Cr}, \mathrm{Ti}, \mathrm{Ca}, \mathrm{Mg}$ ) are strongly underabundant (e.g. van Winckel et al. 1992). Mathis \& Lamers (1992) have suggested that the low metal abundance is not primordial, but is the result of selective depletion of certain metals due to the formation of dust grains. The gas and dust are separated in the circumstellar environment, for which Mathis \& Lamers suggest either the dusty AGB wind, or an accretion disc in the case of a binary system. Clean gas is accreted on to the photosphere, while the dust grains containing the metals are pushed out by stellar radiation pressure. Waters et al. (1992) point out that 4 out of 5 stars with extreme Fe abundance are in a (wide) binary system, and propose that the clean gas was accreted from a circum-system disc rather than fallen back from the AGB wind. 


\section{6. $\mathrm{H} \alpha$ emission}

Almost all post-AGB candidates show some degree of emission in $\mathrm{H} \alpha$. This (variable) emission usually has the shape of a shell profile, i.e. a Violet and Red emission peak combined with a deep central absorption. Some stars show a P Cygni profile (HD 101584; SAO173329; see Waters et al. 1992), while HR 4049 shows alternating P Cygni and shell profiles (Waelkens et al. 1991). The study of Tamura (1992) has shown that in HD 56126 the $\mathrm{H} \alpha$ shell profile changes shape and strength on timescales of minutes. In the stars that show P Cygni profiles an estimate can be made of the outflow velocity, which in most cases is several hundred $\mathrm{km} / \mathrm{s}$, of the order of the surface escape velocity (assuming a mass of $0.6 \mathrm{M}_{\odot}$ ).

The shell profiles have several possible explanations: a disc around the star, infall of material, or pulsation. In most cases pulsation is a likely explanation. The RV variations indicate that many stars indeed show evidence for low-amplitude pulsations. Such pulsations will cause shock waves in the outer atmosphere that will ionize part of the gas, resulting in $\mathrm{H} \alpha$ emission. The shell profiles in post-AGB stars look remarkably similar to the $\mathrm{H} \alpha$ emission seen in RV Tau variables (Lèbre \& Gillet 1991, 1992), although the emission is much stronger in the latter. The difference in line strength may be due to the difference in pulsation amplitude, which can be several tens of $\mathrm{km} / \mathrm{s}$ in RV Tau stars, but is usually less than $10 \mathrm{~km} / \mathrm{s}$ in the $\mathrm{F}$ supergiants.

\section{References}

Arrelano-Ferro, A.: 1984, PASP 96, 641

Bond, H.E.: 1991, in 'Evolution of stars: the photospheric abundance connection', IAU Symp. 145, Eds. G. Michaud \& A. Tutukov, p. 341

Conlon, E.S., Dufton, P.L., Keenan, F.P., McCausland, R.J.H.: 1991, MNRAS 248, 820

Conlon, E.S., 1992, Contributed paper (this volume)

de Boer, K.S., Moehler, S., Theissen, A.: 1992, in proc. of a workshop on post-A GB stars, Leuven, October 1991, ed. C. Waelkens, Leuven University Press (in press)

Garcia-Lario, P., Manchado, A., Pottasch, S.R., Suso, J., Ollig, R.: 1989, in in "From Mira's to Planetary Nebulae: Which Path for Stellar Evolution?", eds. M.O. Mennessier and A. Omont, Editions Frontieres, page 474

Garcia-Lario, P., Manchado, A., Sahu, K.C., Pottasch, S.R.: 1992, A\&A Lett. (in press)

Garcia-Lario, P.: 1991, Ph. D. Thesis, University of La Laguna

Hrivnak, B.J., Kwok, S., Volk, K.M.: 1988, ApJ 331, 832

Hrivnak, B.J., Kwok, S., Volk, K.M.: 1989, ApJ 346, 265

Hu, J.Y., Slijkhuis, S., de Jong, T., 1992

Kwok, S., Hrivnak, B.J., Boreiko, R.T.: 1987, ApJ 312, 303

Kwok, S., 1992, Review paper, (this volume)

Lamers, H.J.G.L.M., Waters, L.B.F.M., Garmany, C.D., Perez, M.R., Waelkens, C.: 1986, A\&A 154, L20

Lèbre, A., Gillet, D.: 1991, A\&A 246, 490

Lèbre, A., Gillet, D.: 1992, A\&A 255, 221

Manchado, A., Garcia-Lario, P., Pottasch, S.R.: 1989a, A\&A 218, 267

Manchado, A., Garcia-Lario, P., Pottasch, S.R.: 1989b, A\&AS 156, 57

Mathis, J.S., Lamers, H.J.G.L.M.: 1992, A\&A 259, L39

Morris, M.; 1989, in "From Mira's to Planetary Nebulae: Which Path for Stellar Evolution?", eds. M.O. Mennessier and A. Omont, Editions Frontieres, page 520

Oudmaijer, R.D., van der Veen, W.E.C.J., Waters, L.B.F.M., Trams, N.R., Waelkens, C., Engelsman, E.: 1993, A\&AS (in press)

Parthasarathy, M., Pottasch, S.R.: 1986, A\&A 154, L16 
Parthasarathy, M., Pottasch, S.R.: 1988, A\&A 203, 117

Pottasch, S.R., Parthasarathy, M.: 1988, A\&A 192, 182

Pottasch, S.R., Bignell, C., Olling, R., Zijlstra, A.A.: 1988, A\&A 205, 248

Quin, D.A., Lamers, H.J.G.L.M.: 1992, A\&A (in press)

Sahu, K.C., Pottasch, S.R., Van De Steene, G., Manchado, A, Garcia-Lario, P.: 1993 (in press)

Schönberner, D.: 1983, ApJ 272, 708

Slijkhuis, S., Hu, J.Y., de Jong, T.: 1991, A\&A 248, 547

Slijkhuis, S.: 1992, Thesis, University of Amsterdam

Stencel, R.E., Backman, D.E.: 1991, ApJS 75, 905

Tamura, S.: 1992, in 'Luminous High Latitude Stars', ed. D.D. Sasselov, ASP Conference Series (in press)

Trams, N.R., Waters, L.B.F.M., Waelkens, C., Lamers, H.J.G.L.M., van der Veen, W.E.C.J.: 1989, A\&A 218, L1

Trams, N.R., Waters, L.B.F.M., Lamers, H.J.G.L.M., Waelkens, C., Geballe, T.R., Thé, P.S.: 1991, A\&AS 87, 361

Trams, N.R., Waters, L.B.F.M., Waelkens, C., Lamers, H.J.G.L.M.: 1992, A\&A (submitted)

van der Veen, W.E.C.J., Habing, H.J., Geballe, T.R.: 1989, A\&A 226, 108

Waelkens, C., Waters, L.B.F.M., Cassatella, A., Le Bertre, T., Lamers, H.J.G.L.M.: 1987, A\&A 181, L5

Waelkens, C., van Winckel, H., Bogaert, E., Trams, N.R.: 1991a, A\&A 251, 495

Waelkens, C., Lamers, H.J.G.L.M., Waters, L.B.F.M., Rufener, F., Trams, N.R., Le Bertre, T., Ferlet, R., Vidal-Madjar, A.: 1991b, A\&A 242, 433

Waelkens, C., Mayor, M.: 1992, in 'Luminous High Latitude Stars', ed. D.D. Sasselov, ASP Conference Series (in press)

Waelkens, C., Waters, L.B.F.M.: 1992, in 'Luminous High Latitude Stars', ed. D.D. Sasselov, ASP Conference Series (in press)

Waters, L.B.F.M., Trams, N.R., Waelkens, C.: 1991, in "The Infrared Spectral Region of Stars", eds. C. Jaschek and Y. Andrillat, Cambridge University Press, p. 40

Waters, L.B.F.M., Waelkens, C., Trams, N.R.: 1992, A\&A 262, L37

Waters, L.B.F.M., Waelkens, C., Mayor, M., Trams, N.R.: 1993, A\&A (in press) 